\title{
Impact of liposome bupivacaine on the adequacy of pain management and patient experiences following aesthetic surgery: Results from an observational study
}

\author{
Michael C Edwards MD ${ }^{1}$, Evan Sorokin MD², Mark Brzezienski MD³ , Farzad R Nahai MD ${ }^{4}$, Richard Scranton MD5 \\ Holly Wall $\mathrm{MD}^{6}$, Simeon Wall $\mathrm{MD}^{6}$, Stephan Finical $\mathrm{MD}^{7}$, Kevin Smith $\mathrm{MD}^{7}$; On behalf of the EXCLAIM Study Group
}

MC Edwards, E Sorokin, M Brzezienski, et al. Impact of liposome bupivacaine on the adequacy of pain management and patient experiences following aesthetic surgery: Results from an observational study. Plast Surg 2015;23(1):15-20.

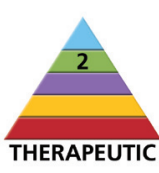

BACKGROUND: Despite the efficacy of opioid analgesics for postsurgical pain, they are associated with side effects that may complicate recovery. Liposome bupivacaine is a prolonged-release formulation of bupivacaine approved for intraoperative administration at the surgical site for postsurgical analgesia.

OBJECTIVES: To evaluate the effect of a single intraoperative administration of liposome bupivacaine on postsurgical pain, opioid use and opioidrelated side effects in subjects undergoing breast surgery and/or abdominoplasty.

METHODS: In the present phase IV, multicentre, prospective observational study, subjects received a single intraoperative administration (266 mg) of liposome bupivacaine. Rescue analgesia was available to all subjects as needed. Outcome measures, assessed through postoperative day 3 , included postsurgical pain intensity (11-point numerical rating scale), opioid consumption and overall benefit of analgesic score. Results were evaluated comparing investigators' previous experience with similar surgeries.

RESULTS: Forty-nine subjects entered the study: 34 underwent breast surgery only and 15 underwent abdominoplasty with or without breast surgery (six underwent breast surgery in addition to abdominoplasty). Mean numerical rating scale pain scores remained $\leq 4.3$ from discharge through postoperative day 3. Median daily oral opioid consumption was approximately 1.0 tablet postoperatively on the day of surgery and was approximately 2.0 tablets by postoperative day 3 . Mean overall benefit of analgesic score ranged between 2.8 and 4.9 throughout the study.

CONCLUSION: In this particular subject population, liposome bupivacaine was associated with low pain intensity scores and reduced opioid consumption compared with the investigators' previous experiences. Subjects' satisfaction with postsurgical analgesia was high, with a low burden of opioid-related side effects.

Key Words: Analgesia; Bupivacaine; Cosmetic surgery; Opioid analgesics; Postoperative pain

$\Delta$ pproximately 1.6 million cosmetic plastic surgery procedures

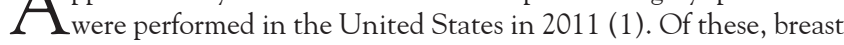
augmentation was the most commonly performed surgery (approximately 307,000 procedures), while abdominoplasty was the sixth most common (approximately 116,000 procedures) (1).

Most patients experience significant pain following surgical procedures, including aesthetic surgeries, with little evidence that the incidence or severity of postsurgical pain has declined in recent decades $(2,3)$. Abdominoplasty, in particular, is considered by plastic surgeons to

\section{Les effets de la bupivacaïne liposomique sur l'efficacité de la gestion de la douleur et des expériences des patients après une chirurgie esthétique : les résultats d'une étude d'observation}

HISTORIQUE : Malgré l'efficacité des opioïdes pour soulager la douleur postchirurgicale, des effets secondaires peuvent compliquer le rétablissement. La bupivacaïne liposomique est une formulation à libération prolongée approuvée pour l'administration peropératoire d'une analgésie postchirurgicale au site opératoire.

OBJECTIFS : Évaluer l'effet de l'administration peropératoire d'une seule dose de bupivacaïne liposomique sur la douleur postchirurgicale, ainsi que sur l'utilisation d'opioïdes et leurs effets secondaires chez des sujets subissant une chirurgie mammaire, une abdominoplastie ou les deux interventions.

MÉTHODOLOGIE : Dans le cadre de la présente étude d'observation prospective et multicentrique de phase IV, les sujets se sont fait administrer une seule dose peropératoire de bupivacaïne liposomique $(266 \mathrm{mg})$. Tous les sujets pouvaient recevoir une analgésie de secours, au besoin. Les mesures des résultats, évaluées jusqu'au troisième jour postopératoire, incluaient l'intensité de la douleur postchirurgicale (sur une échelle numérique de onze points), la consommation d'opioïdes et les bienfaits globaux du score analgésique. Les chercheurs ont évalué les résultats en les comparant à leur expérience de chirurgies similaires.

RÉSULTATS : Quarante-neuf sujets ont participé à l'étude : 34 ont subi seulement une chirurgie mammaire et 15, une abdominoplastie accompagnée ou non d'une chirurgie mammaire (six ont subi une chirurgie mammaire en plus de l'abdominoplastie). Les scores de douleur moyens sur l'échelle numérique ne dépassaient pas 4,3 entre le congé et le troisième jour postopératoire. La consommation quotidienne médiane d'opioïdes par voie orale après l'opération était d'environ 1,0 comprimé le jour de la chirurgie et d'environ 2,0 comprimés le troisième jour postopératoire. Les avantages globaux moyens du score analgésique se situaient entre 2,8 et 4,9 tout au long de l'étude.

CONCLUSION : Au sein de cette population de sujets, la bupivacaïne liposomique s'associait à de faibles scores d'intensité de la douleur et à une consommation réduite d'opioïdes par rapport aux expériences passées des chercheurs. Les sujets étaient très satisfaits de l'analgésie postchirurgicale et présentaient un faible fardeau d'effets secondaires liés aux opioïdes.

be one of the most painful cosmetic procedures. Fear of postsurgical pain is a prominent concern for most patients about to undergo surgery, and is one of the principal reasons that patients postpone or avoid surgery. In addition, there is reluctance among some surgeons regarding initiating a discussion with patients about postsurgical pain for fear of increasing patients' anxiety or influencing the decision to undergo elective aesthetic surgery.

Achieving adequate postsurgical pain management and minimizing analgesic-related adverse events (AEs) during the first two to three

${ }^{1}$ Edwards Plastic Surgery, Las Vegas, Nevada; ${ }^{2}$ Delaware Valley Plastic Surgery, Cherry Hill, New Jersey; ${ }^{3}$ Plastic Surgery Group, Chattanooga,

Tennessee; ${ }^{4}$ Paces Plastic Surgery, Atlanta, Georgia; ${ }^{5}$ Pacira Pharmaceuticals, Inc, Parsippany, New Jersey; ${ }^{6}$ The Wall Center for Plastic

Surgery, Shreveport, Louisiana; ${ }^{7}$ Charlotte Plastic Surgery, Charlotte, North Carolina, USA

Correspondence: Dr Stephan Finical, Charlotte Plastic Surgery, 2215 Randolph Road, Charlotte, North Carolina 28207, USA.

Telephone 704-372-6846, fax 704-342-0752, e-mail sfinical@charlotteplasticsurgery.com 
postsurgical days are important factors for timely ambulation and recovery, as well as for patients' satisfaction regarding their surgical experience (4-7). Although opioid analgesics are effective and are a well-established mainstay of postsurgical pain management, opioidrelated AEs are common and often result in negative clinical outcomes and increased health care-related costs $(4,8)$. For these reasons, the use of multimodal analgesic regimens, which use a range of different types of analgesics and administration routes, is strongly recommended by the American Society of Anesthesiologists (6).

Local anesthetics have a prominent place in most multimodal analgesic regimens in surgical settings. Several studies have assessed the utility of elastomeric pain pumps for delivery of local anesthetics to prolong postsurgical analgesia in subjects undergoing breast surgery (9-13) and abdominoplasty $(14,15)$. However, results regarding analgesic efficacy and opioid use with regimens involving a pump versus no-pump regimens have been mixed. There have been several reports of pump-related safety issues, such as inconsistent infusion-rate accuracy associated with variability in body temperature, location of sensorfluid restrictors and fill volume (16,17). Also, clogging and/or leaking of the pump catheter, inadvertent catheter disconnection, premature emptying of the medication reservoir and pump failure due to technical problems have all been reported in the literature (18-20).

In 2011, a multivesicular liposomal formulation of the local anesthetic bupivacaine (bupivacaine liposome injectable suspension, Exparel, Pacira Pharmaceuticals, Inc, USA) was approved for administration into surgical sites to produce postsurgical analgesia (21). Liposome bupivacaine is formulated to allow gradual release of bupivacaine over time, following a single administration, to produce extended analgesia (21).

The present study evaluated a single intraoperative administration of liposome bupivacaine $(266 \mathrm{mg}$ ) in subjects undergoing elective abdominoplasty, breast augmentation or breast reduction surgery. Outcomes of interest included severity of postsurgical pain, amount of postsurgical opioids used, burden of opioid-related AEs and subject satisfaction with postsurgical analgesia.

\section{METHODS}

The Assessing the Impact of EXPAREL On Patient OutComes and Ease Of Use When Administered By InfiLtration In Subjects Undergoing Breast AugmentatIon Mammoplasty, Breast Reduction and Abdominoplasty Surgeries (EXCLAIM) study was a phase IV, multicentre, prospective, observational study designed to generate outcome data with respect to postsurgical analgesia following a single intraoperative administration of liposome bupivacaine $(266 \mathrm{mg})$ into the surgical site in subjects undergoing breast augmentation mammoplasty, breast reduction and/or abdominoplasty.

Each study site obtained the approval of the appropriate institutional review board/independent ethics committee and conducted the study in accordance with International Conference on Harmonisation Good Clinical Practice guidelines and/or United States Food and Drug Administration Title 21 of the Code of Federal Regulations Part 56 and the Declaration of Helsinki. Written informed consent was obtained from all subjects before their enrollment in the present study.

Nonpregnant women $\geq 18$ years of age undergoing breast augmentation mammoplasty, breast reduction and/or abdominoplasty, with American Society of Anesthesiologists physical status classification of 1 to 3, were eligible to participate. Men undergoing abdominoplasty were also eligible for study participation. Exclusion criteria included history of hypersensitivity or allergic reactions to local anesthetics, chronic use of opioids before study entry and any condition that, in the investigator's opinion, would preclude or be a potential contraindication to study participation.

Before surgery, subject eligibility was assessed, medical history was recorded, a pregnancy test was conducted and baseline pain intensity at rest was assessed using an 11-point numerical rating scale (NRS; $0=$ no pain, 10 = worst possible pain). Surgeries were performed according to each surgeon's usual techniques. Abdominoplasty procedures were conducted with plication; for augmentation mammoplasty surgeries, incisions were made at the mammary fold and breast implants were placed submuscularly. Short-acting opioids (eg, fentanyl and/or sufentanil) were administered intraoperatively according to the surgeon's usual practice. Before surgical closure, liposome bupivacaine (266 mg) was administered via infiltration into the surgical site. For breast surgeries, liposome bupivacaine $(266 \mathrm{mg}$ ) was given undiluted $(20 \mathrm{~mL})$ or diluted with $0.9 \%$ saline to a total volume of up to $100 \mathrm{~mL}$ (dilution volume was at the investigator's discretion) and administered in equal volumes to the left and right breast surgical sites according to the surgeon's normal practice. For abdominoplasty, liposome bupivacaine (266 mg, $20 \mathrm{~mL}$ ) was diluted to a volume of $40 \mathrm{~mL}$ to $100 \mathrm{~mL}$ (at the investigator's discretion) and administered to the surgical site according to the surgeon's normal practice. For subjects who underwent concomitant abdominoplasty and breast surgery, dilution volume and amount of study drug administered across surgical sites was left to the discretion of the surgeon. For all surgeries, the duration of surgery and the date/time of surgical closure were recorded.

Following surgery, pain intensity (NRS) was assessed when the subject awoke and then hourly until discharge. The type, dosage and time of administration of any postsurgical analgesics were recorded until discharge. Acetaminophen and/or nonsteroidal anti-inflammatory drugs were administered as needed for minor pain (NRS score $\leq 4$ ). For moderate to severe pain (NRS score $>4$ ), intravenous morphine sulfate, hydromorphone hydrochloride and/or orally administered opioid combinations were given as needed until subject discharge. Analgesic consumption was recorded postoperatively on the day of surgery. In addition, each subject completed an overall benefit analgesic score (OBAS) assessment daily from day of surgery through postoperative day (POD) 3. The OBAS questionnaire is a validated, multidimensional tool that assesses pain intensity, opioid-related AEs and subject satisfaction (22). It includes questions on the following items: pain intensity (scale: 0 = minimal pain; 4 = maximal pain), opioid-associated AEs (vomiting, itchiness, sweating, freezing and dizziness; scale: $0=$ not at all; 4 = very much) and overall subject satisfaction with pain treatment (scale: $0=$ not at all; $4=$ very much). The overall OBAS score is derived by summing the scores for questions 1 through 6 and adding " 4 minus the question 7 score." In addition, a single-question assessment of the impact (of pain) on normal daily activities (IONDA; "On a scale of 0 to 10 , please enter the number that best describes the impact of your surgical pain on normal daily activity") was administered on the day of surgery and POD 1, POD 2 and POD 3 (scale: 0 = none, I am doing everything I did before surgery; 10 = I am not able to do any activities I did before surgery).

Following discharge from the surgical facility, nonsteroidal antiinflammatory drugs and/or acetaminophen (maximum dose $4000 \mathrm{mg}$ daily) were prescribed for pain, along with opioid medications according to the investigator's usual practice. Any calls or unscheduled office visits to the surgeon's office, along with the reason(s) (eg, inadequate pain control, nausea/vomiting, pruritus) for the call/visit, were recorded. From the day of surgery through the postsurgical office follow-up visit (on POD 5 to POD 7), pain intensity (NRS) was recorded daily at 09:00; analgesic consumption was recorded and the OBAS was completed daily at 21:00.

Outcome measures included postsurgical pain intensity (NRS) and OBAS through POD 3, total volume of liposome bupivacaine plus diluent administered, duration of surgery, duration of stay in the postanesthesia care unit, time of first postsurgical opioid use, total postsurgical opioid use (expressed as the number of opioid analgesic tablets), number and purpose of postsurgical subject calls and/or unscheduled visits to the surgeon's office or surgical centre, and postsurgical AEs observed/reported through end of study (POD 3 ).

All data were summarized using descriptive statistics, stratified by type of surgery. For analysis purposes, the breast surgery group included subjects who underwent breast augmentation or breast reduction procedures. The abdominoplasty with or without breast surgery group included subjects for whom abdominoplasty was the 
TABLE 1

Demographics and subject characteristics

\begin{tabular}{lcc}
\hline Parameter & $\begin{array}{c}\text { Abdominoplasty } \pm \\
\text { breast surgery }(\mathbf{n = 1 5})\end{array}$ & $\begin{array}{c}\text { Breast surgery* } \\
(\mathbf{n}=\mathbf{3 4})\end{array}$ \\
\hline Age, years, mean \pm SD & $43 \pm 13$ & $33 \pm 10$ \\
Sex & 14 & 34 \\
$\quad$ Female & 1 & 0 \\
Male & 13 & 26 \\
Race & 1 & 3 \\
White & 1 & 5 \\
Black & $168 \pm 10$ & $163 \pm 8$ \\
Other & $74 \pm 15$ & $61 \pm 10$ \\
Height, cm, mean \pm SD & 0 & 0 \\
Weight, kg, mean \pm SD & $1.2 \pm 0.4$ & $1.2 \pm 0.4$ \\
Recent history of opioid use ${ }^{\dagger}$ & & \\
ASA physical status & & \\
classification, mean \pm SD & & \\
\hline
\end{tabular}

Data presented as $n$ unless otherwise specified. *Thirty-one subjects had breast augmentation and three subjects had breast reduction surgery; ${ }^{\dagger}$ History of opioid use within three months before the current surgery. ASA American Society of Anesthesiologists

primary surgical procedure, whether they also underwent breast surgery. The amount of opioids administered after surgery was expressed as the number of tablets.

\section{RESULTS}

A total of 49 subjects were included in the study: 34 in the breast surgery group and 15 in the abdominoplasty with or without breast surgery group; all subjects were female except one (a male abdominoplasty subject was included). Subject demographic information and baseline characteristics are summarized in Table 1. Most subjects (39 of 49) were white and all were opioid-naïve. In the abdominoplasty with or without breast surgery group, six of 15 subjects underwent both abdominoplasty and breast augmentation during the same surgery, while the remainder (nine of 15 ) underwent abdominoplasty only. Of the 34 subjects in the breast surgery group, 31 underwent breast augmentation and three underwent breast reduction.

Mean $( \pm$ SD) duration of surgery was $175 \pm 80 \mathrm{~min}$ in the abdominoplasty with or without breast surgery group (median $129 \mathrm{~min}$ ) and $81 \pm 50 \mathrm{~min}$ in the breast surgery group (median $66 \mathrm{~min}$ ). Mean total volume of bupivacaine infused was $67 \pm 18 \mathrm{~mL}$ in the abdominoplasty with or without breast surgery group (median $70 \mathrm{~mL}$ ) and $50 \pm 25 \mathrm{~mL}$ in the breast surgery group (median $40 \mathrm{~mL}$ ). Mean duration of postanesthesia care unit stay was $97 \pm 42 \mathrm{~min}$ in the abdominoplasty with or without breast surgery group (median $75 \mathrm{~min}$ ) and $80 \pm 28 \mathrm{~min}$ in the breast surgery group (median $77 \mathrm{~min}$ ).

In both surgery groups, mean NRS pain intensity scores remained $\leq 4.3$ each day from discharge on the day of surgery through POD 3 (Figure 1). Median NRS pain intensity scores at discharge, POD 1, POD 2 and POD 3 were 5.0, 5.0, 4.0 and 3.0, respectively, in the abdominoplasty with or without breast surgery group; and 3.5, 3.0, 3.0 and 3.0, respectively, in the breast surgery group. The proportion of subjects who reported mild pain (NRS score $\leq 4)$ each postsurgical day through POD 3 ranged from seven of $15(47 \%)$ on POD 1 in the abdominoplasty with or without breast surgery group to 28 of 34 (82\%) on POD 3 in the breast surgery group (Figure 2).

The median daily consumption of opioid analgesic tablets for each surgery group from day of surgery through POD 3 is illustrated in Figure 3; in both groups, median opioid consumption was highest on POD 1 and steadily declined through POD 3. The number of subjects who remained opioid-free and the number who received $\leq 2$ opioid tablets per day through POD 3 are illustrated in Figure 4.

Mean total OBAS data are summarized in Figure 5, with scores for each of the seven OBAS domains summarized in Table 2. Total OBAS remained low throughout the study, indicating satisfaction with analgesia

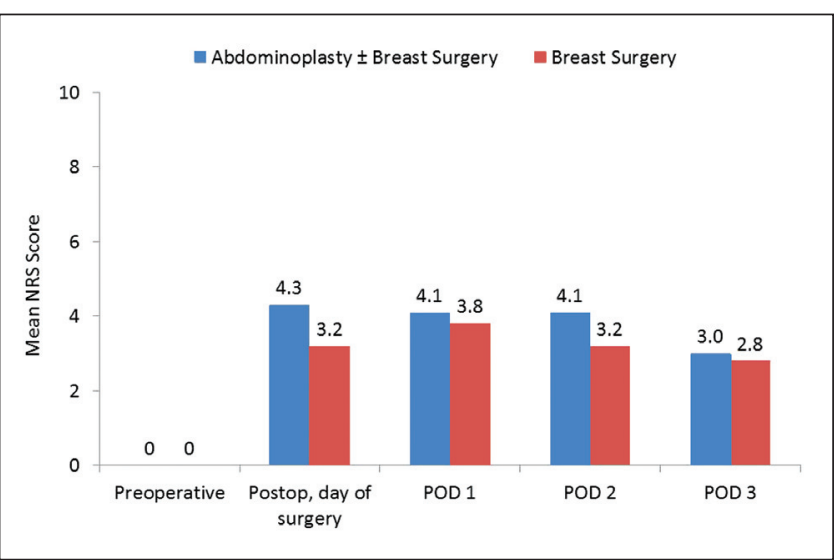

Figure 1) Mean pain intensity scores, stratified according to treatment group from discharge through postoperative day (POD) 3 using the 11-point numerical rating scale (NRS) in which $0=$ no pain and $10=$ worst possible pain

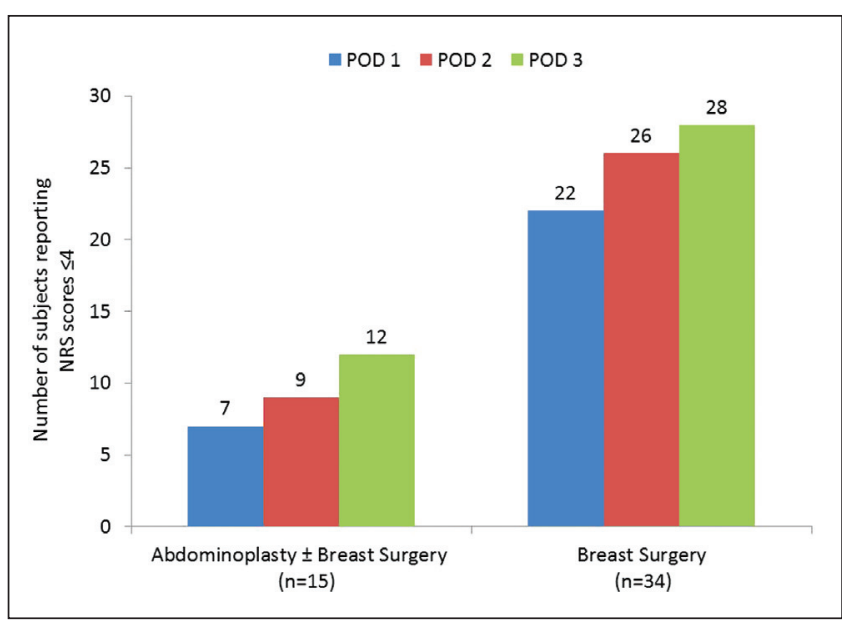

Figure 2) Number of subjects in each treatment group who reported mild pain on postoperative days (PODs) 1,2 and 3. Mild pain was defined as a score $\leq 4$ on the numerical rating scale (NRS), in which $0=$ no pain and $10=$ worst possible pain

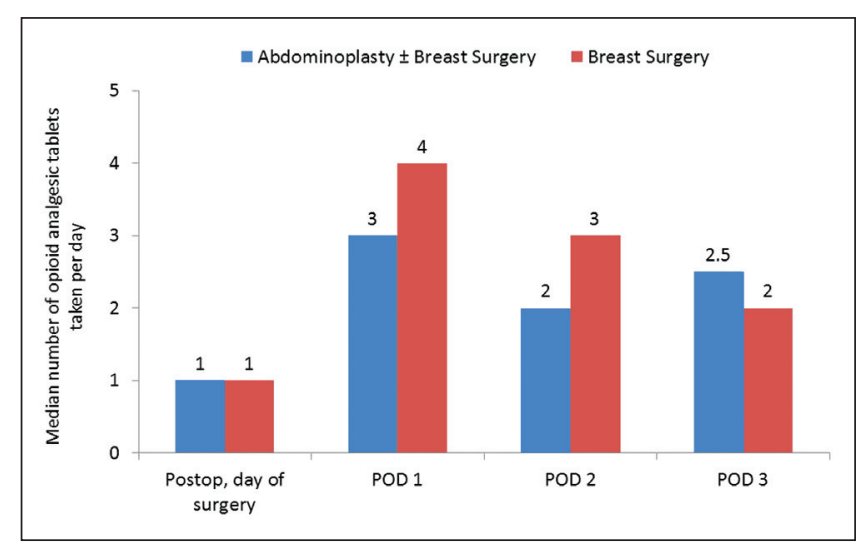

Figure 3) Median daily opioid consumption (number of tablets) from discharge through postoperative day (POD) 3, stratified according to treatment group

and a low burden of opioid-associated side effects. Individual mean domain scores for pain intensity and opioid-associated side effects were low throughout the study, and mean scores for the "Satisfaction with Pain Management" domain were $>3$ throughout the study, with a 


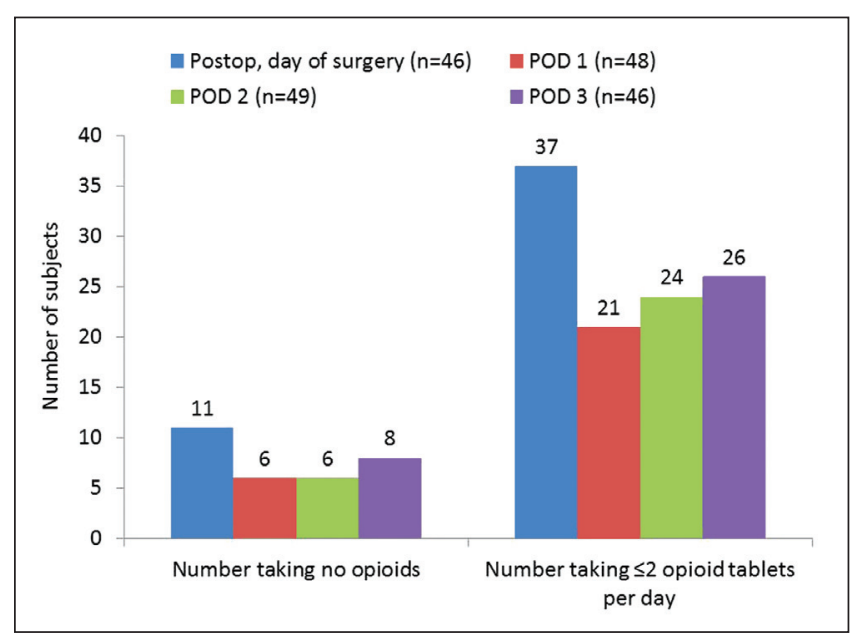

Figure 4) Number of subjects in the overall study population who met thresholds for low opioid consumption according to day, from discharge through postoperative day (POD) 3

single exception (on POD 1 in the abdominoplasty with or without breast surgery group).

Mean ( \pm SD) IONDA scores at discharge were $6.9 \pm 2.5$ in the breast surgery group and 7.1 \pm 3.0 in the abdominoplasty with or without breast surgery group. In the breast surgery group, mean IONDA scores declined to $5.2 \pm 2.4$ by POD 3. In the abdominoplasty with or without breast surgery group, the mean IONDA score remained $>7$ each day after surgery, and was $7.7 \pm 1.6$ on POD 3 .

A total of three subjects, all in the breast surgery group $(n=34)$, made a phone call or unscheduled visit to the hospital or surgeon's office following discharge from the surgical facility. All three calls/ visits were related to postsurgical pain after breast augmentation mammoplasty; one was from a pharmacy related to a potential drug tolerance issue based upon the subject's previous medication history. One of these subjects did not have an NRS pain intensity score at discharge but had a pain severity (domain 1) OBAS of 1 at discharge. For the other two subjects, NRS pain intensity scores at discharge were 1 and 4 , respectively; both had a pain severity OBAS of 2 at discharge. No subjects in the abdominoplasty with or without breast surgery group had unscheduled visits after surgery.

\section{DISCUSSION}

The present prospective, observational study evaluated the addition of a single intraoperative administration of liposome bupivacaine (266 mg) to usual analgesic therapy in subjects undergoing cosmetic plastic surgery procedures at established surgical practices. The surgery types selected for the study (breast augmentation, breast reduction and abdominoplasty) are associated with significant postsurgical pain and consequent risk of opioid-related AEs.

From discharge through POD 3, mean and median NRS pain intensity scores were consistently reflective of low to moderate pain intensity across both surgery groups. Scores for the abdominoplasty with or without breast surgery group were generally slightly higher than for the breast surgery group, consistent with the investigators' experience that abdominoplasty is typically a more painful procedure than breast surgery, although the between-group differences decreased with time after surgery (Figure 1).

Postsurgical consumption of opioid analgesics was also low, with the median daily dose peaking at approximately three to four tablets/day on POD 1 and declining to approximately two to three tablets/day on PODs 2 and 3. These amounts are substantially lower than the amount of postsurgical opioids typically prescribed in the investigators' practices (approximately six to 12 tablets/day). The amount of opioids consumed was similar between surgical procedure groups, a somewhat unexpected finding given the investigators' experience (supported by pain intensity

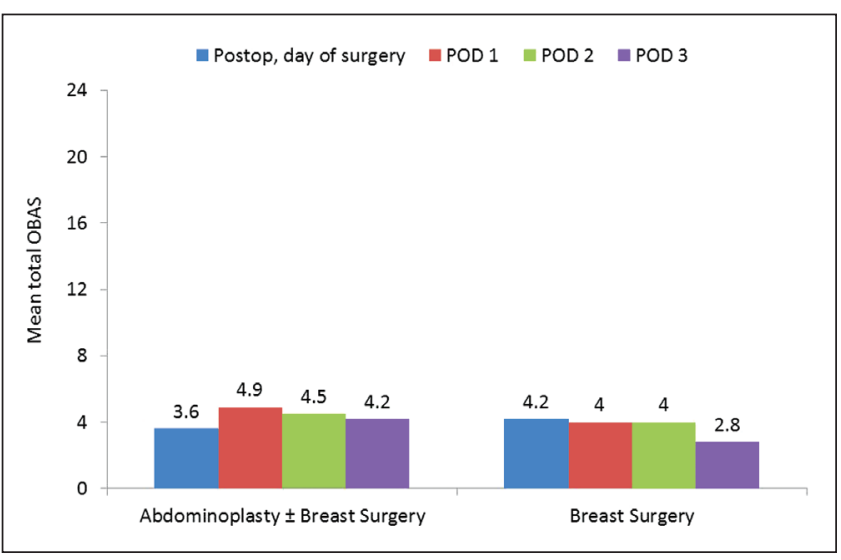

Figure 5) Mean total overall benefit of analgesic score (OBAS), stratified according to treatment group, according to day, from discharge through postoperative day (POD) 3. Maximum score is 28. Higher scores are indicative of poor overall benefit of analgesia

scores in this study) that abdominoplasty is typically a more painful procedure than breast augmentation or breast reduction surgery.

The combination of relatively low pain scores and low opioid consumption observed in the present study is particularly encouraging, because most subjects in the study were undergoing surgery for the first time. In the experience of the investigators, surgery-naive subjects typically expect that postsurgical pain will be severe.

The typical discharge protocol following cosmetic surgery in the investigators' practices involves discharging patients on the same day as surgery, with a prescription for a four- to five-day opioid analgesic regimen. The use of Web-based electronic subject diaries allowed the surgeons to accomplish early subject discharge and still obtain timely information and subject feedback regarding subject experiences with pain after discharge.

To our knowledge, this is the first published report of results from a prospective study evaluating the efficacy of liposome bupivacaine in subjects undergoing abdominoplasty. Smoot et al (23) recently reported results from their study comparing liposome bupivacaine and bupivacaine hydrochloride for postsurgical analgesia in subjects undergoing augmentation mammoplasty. It is difficult to compare our results with those observed by Smoot et al (23) because the dose of liposome bupivacaine evaluated in the Smoot study was two times higher than the dosage used in our study, which is the maximum United States Food and Drug Administration-approved dose of liposome bupivacaine.

There are several limitations of the present study. First, this was an observational, open-label design with a relatively small study population. Second, the study protocol allowed for flexibility in investigators' chosen method of study drug administration. While this procedural flexibility could be considered a confounding factor, it also allowed for a clinically meaningful evaluation of liposome bupivacaine. Investigators were able to easily incorporate the study drug into their usual procedures without having to follow a specific protocol for administration, which enabled them to evaluate the study drug under conditions approximating 'real world' clinical use. While the dilution of study drug was allowed to vary based on the surgeon's preference and size of the surgical site, the amount of study drug administered (266 mg) remained consistent among the investigators. Third, because this was a single-arm observational study, cost-related assessments were not included in the protocol. Although the product cost of liposome bupivacaine is higher than some other commonly used analgesics for perioperative pain (eg, opioids, standard formulations of local anesthetics), in our experience, the cost of liposome bupivacaine is typically offset by improved outcomes, such as better pain relief, less opioid consumption and shorter length of hospital stay observed in subjects treated with this agent. 


\begin{tabular}{|c|c|c|c|c|c|c|c|}
\hline \multirow[b]{2}{*}{ Time since surgery } & \multicolumn{7}{|c|}{ OBAS domains* } \\
\hline & Pain & Vomiting & Itching & Sweating & Freezing & Dizziness & $\begin{array}{c}\text { Satisfaction } \\
\text { with pain } \\
\text { management }\end{array}$ \\
\hline \multicolumn{8}{|l|}{ Immediately postsurgery } \\
\hline Abdominoplasty \pm breast surgery $(n=14)$ & $1.3 \pm 0.8$ & $0.1 \pm 0.5$ & $0.6 \pm 1.0$ & $0.2 \pm 0.4$ & $0.5 \pm 0.9$ & $0.5 \pm 0.7$ & $3.6 \pm 0.5$ \\
\hline Breast surgery $(n=32)$ & $1.4 \pm 0.9$ & $0.2 \pm 0.6$ & $0.4 \pm 0.8$ & $0.3 \pm 0.5$ & $0.3 \pm 0.6$ & $0.7 \pm 0.9$ & $3.1 \pm 0.9$ \\
\hline \multicolumn{8}{|l|}{ Postoperative day 1} \\
\hline Abdominoplasty \pm breast surgery $(n=15)$ & $2.1 \pm 1.0$ & $0.1 \pm 0.3$ & $0.5 \pm 0.9$ & $0.2 \pm 0.4$ & $0.3 \pm 0.7$ & $0.5 \pm 0.8$ & $2.8 \pm 1.0$ \\
\hline Breast surgery $(n=33)$ & $1.4 \pm 0.8$ & $0.2 \pm 0.8$ & $0.6 \pm 1.1$ & $0.2 \pm 0.4$ & $0.2 \pm 0.5$ & $0.6 \pm 1.0$ & $3.3 \pm 0.7$ \\
\hline \multicolumn{8}{|l|}{ Postoperative day 2} \\
\hline Abdominoplasty \pm breast surgery $(n=15)$ & $1.4 \pm 0.7$ & $0.1 \pm 0.3$ & $1.1 \pm 1.2$ & $0.4 \pm 0.6$ & $0.3 \pm 0.8$ & $0.4 \pm 0.8$ & $3.1 \pm 0.9$ \\
\hline Breast surgery $(n=34)$ & $1.4 \pm 0.9$ & $0.1 \pm 0.4$ & $0.8 \pm 1.1$ & $0.1 \pm 0.4$ & $0.2 \pm 0.5$ & $0.6 \pm 1.0$ & $3.2 \pm 0.8$ \\
\hline \multicolumn{8}{|l|}{ Postoperative day 3} \\
\hline Abdominoplasty \pm breast surgery $(n=14)$ & $1.4 \pm 1.2$ & $0.1 \pm 0.3$ & $1.4 \pm 1.3$ & $0.1 \pm 0.4$ & $0.1 \pm 0.4$ & $0.4 \pm 0.9$ & $3.4 \pm 0.9$ \\
\hline Breast surgery $(n=32)$ & $0.9 \pm 0.9$ & $0.1 \pm 0.3$ & $0.6 \pm 0.8$ & $0.2 \pm 0.6$ & $0.2 \pm 0.6$ & $0.3 \pm 0.7$ & $3.5 \pm 0.8$ \\
\hline
\end{tabular}

Data presented as mean \pm SD. ${ }^{*}$ The five-point scales for the domains are as follows: Pain: $0=$ minimal pain, $4=$ maximum imaginable pain; Vomiting, Itching, Sweating, Freezing and Dizziness (regarding distress and bother from symptoms): $0=$ not at all, $4=$ very much; Satisfaction with pain management: $0=$ not at all, $4=$ very much

\section{CONCLUSION}

The present prospective, observational study of intraoperatively administered liposome bupivacaine showed that subjects undergoing elective abdominoplasty, breast augmentation and breast reduction surgery experienced less postsurgical pain and required lower amounts of postsurgical opioid analgesics than the investigators (all experienced cosmetic surgeons) have observed historically in subjects not receiving liposome bupivacaine.

THE EXCLAIM STUDY GROUP IS COMPRISED OF: Richard Baxter MD; Mark Brzezienski MD; Andrew Cohen MD; Michael C Edwards MD; Stephan Finical MD; Thomas Horn MD; Farzad R Nahai MD; Kevin Smith MD; John D Smoot MD; Evan Sorokin MD; Holly Casey Wall MD; Simeon Wall MD

ACKNOWLEDGEMENTS: This study was funded by Pacira Pharmaceuticals, Inc, which contributed to the study design, statistical analysis, manuscript preparation and subject recruitment costs for the studies. Editorial assistance was provided by Peloton Advantage, LLC, and supported by Pacira Pharmaceuticals, Inc. The authors were fully responsible for the content, editorial decisions and opinions expressed in the current article. No author received an honorarium related to the development of this article.

DISCLOSURES: Dr Edwards is an investigator for Allergan, Inc; Mentor Corporation, Sientra, Inc; and Pacira Pharmaceuticals, Inc, and has received financial support from Pacira Pharmaceuticals, Inc for study participation. He has also received financial support from TouchMD as an advisor and is a stockholder in www.ExplainMySurgery.com. He has no additional financial disclosures to report.

FUNDING: Dr Sorokin has received financial support from Pacira Pharmaceuticals, Inc as a study investigator. Dr Brzezienski has received financial support from Pacira Pharmaceuticals, Inc as a study investigator. Dr Nahai has received financial support from Pacira Pharmaceuticals, Inc as a study investigator. He has no financial interest in Pacira Pharmaceuticals, Inc or Exparel. He has no other disclosures pertinent to this study. Dr Scranton is an employee of Pacira Pharmaceuticals, Inc. Dr H Wall is a partial owner in Surgical Suites at the Wall Center in Shreveport, Louisiana, which received financial support from Pacira Pharmaceuticals, Inc, for research coordination. She has no other disclosures pertinent to this study. Dr S Wall is a partial owner in Surgical
Suites at the Wall Center in Shreveport, Louisiana, which received finacial support from Pacira Pharmaceuticals, Inc, for rsearch coordination. He has no other disclosures pertinent to this study. Dr Finical has received financial support from Pacira Pharmaceuticals, Inc, as a study investigator and from Allergan, Inc, as a consultant. Dr Smith is a shareholder of Pacira Pharmaceuticals, Inc, and has received financial support from Pacira Pharmaceuticals, Inc and SkinMedica, an Allergan Company, as a consultant.

\section{REFERENCES}

1. American Society of Plastic Surgeons. American Society of Plastic Surgeons website. 2011 Plastic Surgery Statistics Report. <www.plasticsurgery.org/News-and-Resources/2011-Statistics-html> (Accessed November 6, 2014).

2. Apfelbaum JL, Chen C, Mehta SS, Gan TJ. Postoperative pain experience: Results from a national survey suggest postoperative pain continues to be undermanaged. Anesth Analg 2003;97:534-40.

3. Gan TJ, Habib AS, White W, Miller T. Postoperative pain continues to be undermanaged [Abst]. Presented at: Annual Fall Pain Meeting and Workshops of the American Society of Regional Anesthesia and Pain Medicine. Miami Beach, November 15 to 18, 2012.

4. Adamson RT, Lew I, Beyzarov E, Amara S, Reitan J. Clinical and economic implications of postsurgical use of opioid therapy. Hosp Pharm 2011:46(6 Suppl 1):S4-S11.

5. Shang AB, Gan TJ. Optimising postoperative pain management in the ambulatory patient. Drugs 2003;63:855-67.

6. Practice guidelines for acute pain management in the perioperative setting: An updated report by the American Society of Anesthesiologists Task Force on Acute Pain Management. Anesthesiology 2012;116:248-73.

7. Tong D, Chung F. Postoperative pain control in ambulatory surgery. Surg Clin North Am 1999;79:401-30.

8. Oderda GM, Said Q, Evans RS, et al. Opioid-related adverse drug events in surgical hospitalizations: Impact on costs and length of stay. Ann Pharmacother 2007;41:400-7.

9. Baroody M, Tameo MN, Dabb RW. Efficacy of the pain pump catheter in immediate autologous breast reconstruction. Plast Reconstr Surg 2004;114:895-8.

10. Heller L, Kowalski AM, Wei C, Butler CE. Prospective, randomized, double-blind trial of local anesthetic infusion and intravenous narcotic patient-controlled anesthesia pump for pain management after free TRAM flap breast reconstruction. Plast Reconstr Surg 2008;122:1010-8.

11. Kryger ZB, Rawlani V, Lu L, Fine NA. Decreased postoperative pain, narcotic, and antiemetic use after breast reduction using a local anesthetic pain pump. Ann Plast Surg 2008;61:147-52. 
12. Losken A, Parris JJ, Douglas TD, Codner MA. Use of the infusion pain pump following transverse rectus abdominis muscle flap breast reconstruction. Ann Plast Surg 2005;54:479-82.

13. Rawlani V, Kryger ZB, Lu L, Fine NA. A local anesthetic pump reduces postoperative pain and narcotic and antiemetic use in breast reconstruction surgery: A randomized controlled trial. Plast Reconstr Surg 2008;122:39-52.

14. Bray DA Jr, Nguyen J, Craig J, Cohen BE, Collins DR Jr. Efficacy of a local anesthetic pain pump in abdominoplasty. Plast Reconstr Surg 2007;119:1054-9.

15. Smith MM, Hovsepian RV, Markarian MK, et al. Continuousinfusion local anesthetic pain pump use and seroma formation with abdominal procedures: Is there a correlation? Plast Reconstr Surg 2008;122:1425-30.

16. Ilfeld BM, Morey TE, Enneking FK. Portable infusion pumps used for continuous regional analgesia: Delivery rate accuracy and consistency. Reg Anesth Pain Med 2003;28:424-32.

17. Birrer KL, Anderson RL, Liu-DeRyke X, Patel KR. Measures to improve safety of an elastomeric infusion system for pain management. Am J Health Syst Pharm 2011;68:1251-5.

18. Pu LL. The use of a pain pump for optimal postoperative pain management. Plast Reconstr Surg 2006;117:2066-9.
19. Institute for Safe Medication Practices (ISMP). ISMP Medication Safety Alert! Process for handling elastomeric pain relief balls (ON-Q PainBuster and others) requires safety improvements. <www.ismp.org/Newsletters/acutecare/articles/20090716.asp> (Accessed November 6, 2014).

20. Ganapathy S, Amendola A, Lichfield R, Fowler PJ, Ling E. Elastomeric pumps for ambulatory patient controlled regional analgesia. Can J Anaesth 2000;47:897-902.

21. Exparel [prescribing information]. Parsippany, NJ: Pacira Pharmaceuticals, Inc, 2014.

22. Lehmann N, Joshi GP, Dirkmann D, et al. Development and longitudinal validation of the overall benefit of analgesia score: A simple multi-dimensional quality assessment instrument. Br J Anaesth 2010;105:511-8.

23. Smoot JD, Bergese SD, Onel E, Williams HT, Hedden W. The efficacy and safety of DepoFoam ${ }^{\circledR}$ bupivacaine in patients undergoing bilateral, cosmetic, submuscular augmentation mammoplasty: A randomized, double-blind, active-control study. Aesthet Surg J 2012;32:69-76. 